\title{
APORTE DEL ECOSOCIALISMO AL ANÁLISIS DE LOS CONFLICTOS SOCIOAMBIENTALES: UNA APROXIMACIÓN AL CASO DE COMUNIDADES MAPUCHE EN CHILE
}

(Ecosocialism contribution to the analysis of environmental conflicts: an approach to the case of Mapuche communities in Chile)

\author{
MARITZA MARÍN-HERRERA ${ }^{1}$ \\ Universidad de Costa Rica. Costa Rica
}

\section{Resumen}

Como aporte al análisis de los conflictos socio-ambientales enfrentados por comunidades mapuche del sur de Chile, ante la presión histórica sobre los territorios ancestrales ocupados por el Estado chileno y declarados como unidades de conservación natural, se realiza una discusión sobre el aporte del ecosocialismo para abordar esta problemática y sus ventajas frente a otras corrientes ambientalistas. Se hace una revisión exploratoria de algunos de los planteamientos de la ecología profunda y la ecología integral, para posteriormente identificar los elementos distintivos relevantes del ecosocialismo para la temática. De la discusión teórica propuesta se desprende la pertinencia del ecosocialismo latinoamericano como punto de partida para la generación de soluciones, que buscan mejorar la calidad de vida de las comunidades indígenas inmersas en conflictos socioambientales generados por la disputa de territorios donde se hace posible su existencia.

Palabras Clave: Ecosocialismo, conflictos socio-ambientales, mapuche.

\section{Abstract}

This paper presents a discussion regarding the relevance of Ecosocialism to the analysis of socio-environmental conflicts. This work is performed in the context of mapuche communities in the south of Chile and the historical pressure over their ancestral territories that have been occupied by the State and declared natural conservation units. It starts highlighting the contribution of Ecosocialism and its advantages over other environmental lines of thought, specifically by comparing it to

${ }^{1}$ Licenciada en Sociología, Universidad de Costa Rica. Candidata a Magíster en Desarrollo a Escala Humana y Economía Ecológica, Universidad Austral de Chile. Docente Escuela de Estudios Generales, Universidad de Costa Rica. Correo electrónico: mmarin72@gmail.com 
Revista de Ciencias Sociales y Humanidades

the main proposals of Deep Ecology and Integral Ecology. From the theoretical considerations, this articule closes with the presentation of Ecosocialism in LatinAmerica and its role in the generation of alternatives to improve quality of life of indigenous communities who are immersed in socio-environmental conflicts in territories where their own existence is defined.

Keywords: Ecosocialism, socio-environmental conflicts, mapuche.

\section{Introducción}

En Chile, ${ }^{2}$ como en otras partes del mundo, el estudio de los "conflictos socio-

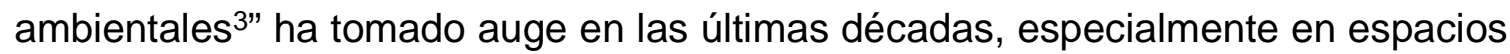
naturales reservados por grupos humanos que mantienen prácticas productivas y de existencia tradicionales, o donde los Estados orientados por una visión ecocentrista han decidido declararlos como unidades de conservación.

En este artículo se revisa el aporte del ecosocialismo al análisis de los conflictos socioambientales, en contraposición a otras corrientes ambientalistas. Para ello, se hace un paso obligado por algunos de los planteamientos de la ecología profunda y la ecología integral, posteriormente se identifica exploratoriamente los elementos distintivos del ecosocialismo que permiten analizar y generar opciones de resolución a este tipo de problemáticas.

Como referente empírico se aborda el caso específico de las comunidades mapuche en Chile que colindan con el sur del Parque Nacional Villarrica, quienes fueron desplazados por el Estado chileno para establecer dicha unidad de conservación,

\footnotetext{
${ }^{2}$ Este artículo se escribe en el marco del trabajo final de graduación que se desarrolló para optar, en diciembre de 2015, por el Magíster en Desarrrollo a Escala Humana y Economía Ecológica de la Universidad Austral de Chile, con el apoyo de la Universidad de Costa Rica.

3 Entenderemos los conflictos socio-ambientales como situaciones donde se da un choque de intereses entre quienes causan un problema ambiental y aquellos que sufren sus consecuencias por el uso de los servicios o bienes ambientales (Del Viso, 2011). Según Leff son "derivados de la desigual distribución ecológica y las estrategias de apropiación de los recursos ecológicos, los bienes naturales y los servicios ambientales" (Leff, 2014, p. 243).
} 
desconociendo la existencia previa de grupos humanos originarios que hacían uso de ese territorio para satisfacer su existencia material y simbólica. En este sentido, la discusión teórica brinda luces sobre formas posibles de reapropiación territorial, que contribuyan a una economía homeostática para las comunidades afectadas, haciendo posible la construcción de una sociedad más justa social, cultural y ambientalmente.

Generar esta discusión, ha sido posible mediante la revisión bibliográfica de los principales representantes, haciendo énfasis en los pensadores del ecosocialismo. Asimismo, se toman extractos de entrevistas a miembros de las comunidades mapuche, realizadas en el marco de una investigación más amplia de la que surge este artículo, que está en una fase exploratoria.

\section{Corrientes ambientalista y conflictos socio-ambientales}

La preocupación creciente por el futuro de la especie humana, ante los embates ambientales provocados por el calentamiento global, se concreta en la construcción de políticas e instituciones globales; también los gobiernos y la sociedad civil tienen una participación activa en la construcción de opciones que permitan la mitigación y la adaptación a los cambios ambientales globales. Paralelamente, el fenómeno llamado globalización conformado por dimensiones políticas, económicas, socioculturales, puede considerarse una fase más avanzada del proyecto modernizante que arrancó en el siglo XVIII y que es acelerado en la segunda mitad del siglo XX, con la construcción del proyecto de desarrollo, fundamentados ambos en el uso de la razón y el dominio de la naturaleza.

La intersección entre un modelo de desarrollo capitalista y las preocupaciones ambientales, se suscitan en el ámbito transnacional mediante discusiones intergubernamentales para las que se genera una parafernalia institucional, con políticas y prácticas que son territorializadas en el espacio local. Es en el ámbito local donde se expresan las contradicciones de una globalización que atenta contra los modos de vida y territorios de comunidades que se suponen pobres y atrasadas (afirmación 
Revista de Ciencias Sociales y Humanidades

respaldada por indicadores cuantitativos y desterritorializados, utilizados por organismos internacionales y gubernamentales), donde las empresas generalmente sin arraigo en los territorios, en conjunto con la intervención estatal inician procesos de desplazamiento, proletarización y homogenización de comunidades y naturalezas ${ }^{4}$.

Históricamente, los países "no desarrollados" han constituido un territorio proveedor de insumos para la industria y el "mejoramiento tecnológico". Pese a ello, existen reservas importantes de recursos, especialmente en aquellos espacios habitados por comunidades tradicionales, entendidas como aquellas que resuelven su cotidianidad haciendo uso pleno de su entorno inmediato y encuentran en sus territorios alimento, medicina, leña, agua, por tanto, su subsistencia biológica depende claramente de su relación con el entorno natural. De igual forma las costumbres y tradiciones que les constituyen como colectivos a lo largo del tiempo están ligados a un "Saber Estar", con frecuencia en equilibrio con la naturaleza.

Sin embargo, nuevos modos de vida irrumpen en lo local expoliando la naturaleza. La capacidad de las comunidades de resolver en sus territorios sus necesidades, es limitada por dos fuentes de presión, una relacionada con el capitalismo y otra con el ambientalismo. La primera tiene relación con el crecimiento ilimitado que plantea el capitalismo y se expresa en la expansión del extractivismo como modo de vida de sociedades que hacen uso de los recursos de todo el planeta, incluyendo los ecosistemas de los que dependen otros grupos sociales. Esta presión se da sobre los bosques, el agua, los minerales, el aire (caso de los sumideros de CO2) convertidos en bienes de mercado.

La segunda, responde a la idea preservacionista de grupos y estados preocupados por la pérdida de biodiversidad y la alerta ante el cambio climático, quienes ven en

${ }^{4}$ Se habla de naturalezas siguiendo a Astrid Ulloa (2013, pág. 122), quien plantea que la visión neoliberal fragmenta la naturaleza para su comercialización (agua, bosques, servicios ecosistémicos). 
la creación y sostenimiento de unidades de conservación la posibilidad de mitigarlos.

\section{a. Ecología profunda fuente de conflictos socioambientales en unidades de conservación}

Los actores sociales que promueven la constitución de unidades de protección, con frecuencia comparten el planteamiento de la ecología profunda ${ }^{5}$. Se establecen espacios naturales sin personas, desplazando a comunidades que dependen de esos espacios para la subsistencia a zonas de mitigación. Aparecen espacios sacralizados a la vez que escindidos de la acción humana. Una crítica a esta posición se encuentra en Gudynas que indica

[...] los componentes sociales de la ecología profunda son débiles.

Fácilmente se caen en actitudes de contemplación e inmovilismo frente a la Naturaleza (con el caso extremo de la creación de los "ecosterios", que son análogos a los monasterios, pero dedicados a la contemplación de la Naturaleza), sin la necesaria reacción ante las situaciones de pobreza, injusticia y marginalidad... (Gudynas, 2003, p. 208).

Bajo esta línea de pensamiento fueron constituidos los parques nacionales conocidos en la modernidad, bajo el modelo estadounidense que tiene como referente el Parque Nacional Yellowstone (1872). Este parque se constituye a partir del interés por proteger la naturaleza de la intervención del ser humano, limitando la expansión de la colonización de tierras en espacios de gran belleza escénica, pero ignorando la presencia previa de grupos humanos que habían convivido

${ }^{5}$ La ecología profunda tiene carácter biocentrista. El ser humano y la naturaleza incluyendo otros animales, tienen la misma jerarquía, al respecto Arne Naess (1973) utiliza la imagen relacional campo-total que no solo disuelve el concepto hombre-en el -medio ambiente, sino también el concepto de toda cosa "en" su medio, de tal forma que el ser humano no posee un estatus superior, aplicando una igualdad ecológica. 
Revista de Ciencias Sociales y Humanidades

ancestralmente con esa naturaleza. En este sentido el historial de los parques nacionales indica que "se establecieron después del desplazamiento o eliminación de los pueblos nativos que vivían en estos territorios" (Martinez-Alier, 2014, p. 30).

El Parque Nacional Villarrica ubicado en la Región de la Araucanía y la Región de Los Ríos en Chile, fue creado el 28 de noviembre de 1940 (Decreto Supremo N²236), como resultado de fusionar la Reserva Forestal del mismo nombre y el Parque Nacional Vicuña Mackena; se establece en los "únicos terrenos de propiedad del Estado que no fueron colonizados por ser marginales para la agricultura, ganadería e incluso para la utilización forestal" (Corporación Nacional Forestal, 2006: p. 8). Si bien la declaratoria sirvió para conservar importantes hectáreas de flora y fauna nativa, invisibilizó la propiedad ancestral del pueblo Mapuche sobre el territorio ahora destinado a parque nacional, y bajo una nueva relación ser humano-naturaleza, se le excluyó de las prácticas culturales asociadas a su relación con el mismo, imponiendo una matriz colonial de poder, "colonizando y subalternizando otras formas de ser, de sentir, de pensar, de hacer, de tejer la vida", tal como afirma Gerrero respecto a este nuevo tipo de relación (Guerrero, 2010, p. 12).

Ejemplo del desplazamiento geográfico y cultural, de las comunidades mapuche respecto al Parque, han sido las veranadas, estas eran un espacio comunitario utilizado en la época estival para el pastoreo de los animales permitiendo el engorde con pastos y frutos de mejor calidad; para este período y práctica los comuneros mapuche, quienes habitaban durante el invierno en zonas menos afectadas por la nieve y el invierno, construían los "sayel" o casas para el veraneo, donde también sociabilizaban con otras familias de la comunidad. Según un adulto mayor de la comunidad, (E.H., comunicación personal, 28 de junio de 2015), su madre le relataba cómo la construcción del "sayel" era comunitaria y espontánea, cada familia le agregaba en su visita algo nuevo, además tenían ahí los utensilios de cocina y todo lo que se necesitaba para habitar por varios días en el lugar, pudiendo disfrutar de al menos un mes de la "biodiversidad" presente en el faldeo del volcán. 
Esta práctica fue erradicada por completo en 1970 por la Corporación Nacional Forestal, ente encargado de administrar las unidades de protección chilenas (Corporación Nacional Forestal, 2006), al respecto Aylwin (Aylwin, 2008) señala que:

[...] la memoria de los ancianos mapuches no olvida hasta ahora como [sic] sus refugios en los faldeos del Ruka Pillán [nombre en Mapusungún del conocido hoy como Volcán Villarrica] fueron quemadas por quienes administraron este $\mathrm{PN}$, ni olvida los juicios seguidos en su contra por la tala de unos pocos árboles para la construcción de los refugios de veranada.

Con su constitución y la separación ser humano y la naturaleza, el Parque Nacional Villarrica pasa a formar parte de lo que Gudynas llamaría "ecosterios", lo cual se concretó en el año 1970 con la erradicación de las veranadas, donde el Estado se apropia de tierras ancestrales y los recursos asociados a la existencia del pueblo Mapuche, dificultando su acceso a estos espacios para satisfacer sus necesidades básicas fundamentales, tanto materiales como simbólicas, pero lo pone a disposición del público para la contemplación de las bellezas naturales.

\section{b. Ecología integral hacia la "modernización" del territorio mapuche}

Así como la constitución misma de un parque nacional o zona de preservación de la naturaleza, genera conflictos socio-ambientales, igualmente lo hace la modernización de estos espacios para incorporarlas a la dinámica del mercado "verde", a través del "turismo" o venta de servicios ecosistémicos, pasando del "culto a lo silvestre" o ecología profunda, al "evangelio de la ecoeficiencia" o ecología integral que "se preocupa por el manejo sustentable y "uso prudente" de los recursos naturales y el control de la contaminación [...] descansando en la creencia de que las nuevas tecnologías y la "internalización de las externalidades" son instrumentos decisivos de la modernización ecológica" (Martinez-Alier, 2014, p. 36). La principal herramienta de esta corriente es la economía ambiental, desde la cual emanan 
concepciones como "servicios ecosistémicos" y soluciones compensatorias como el pago de servicios ambientales.

Para las comunidades mapuche desplazadas para establecer el Parque Nacional Villarrica, el enfoque de la ecología integral constituye un riesgo en tanto, la modernización de esta área busca atraer recursos económicos para el "desarrollo" de las zonas de amortiguamiento, mediante el aumento de la visitación turística al parque. Para esto han planteado en diferentes áreas, figuras de concesión privadas para la construcción y mejoramiento de la infraestructura, insertándose en la ecuación del conflicto los intereses de privados y transnacionales del turismo. Igualmente, se plantean "mejoras" en la vialidad, de tal forma que se conectan diferentes destinos turísticos (Gobierno de Chile, 2013), aquí nuevamente el interés de los privados a quienes se les concesionará la carretera, promueven proyectos tendientes al "gigantismo" que les genere mejores dividendos. Todos estos proyectos tienen sustento en la ecoeficiencia y son sometidos a las leyes del mercado, en tanto, hay una apuesta a la atracción turística como medio para financiar la conservación.

Una de las consecuencias de la "economía verde" presentada en este tipo de proyecto, es la generación de fracturas y conflictos a lo interno de las comunidades, que además se caracterizan por ser interculturales; tanto los mapuche como los no mapuche o colonos entran en discusión por la definición de un proyecto de "desarrollo" que busca territorializarse, desde el ámbito internacional y nacional al local. Es así como una lideresa de la comunidad de Traitraico 6 ante la pavimentación de la carretera que conduce al Parque Nacional Villarrica, manifiesta "qué rico caminar en la tierra, yo vivo diferente... me muevo caminando o a caballo, ya con carretera tengo que buscar otro camino, los autos van a pasar muy rápido, eso es peligroso" (I.C., comunicación personal, 10 de diciembre de 2014). Sin embargo, los

6 Traitraico es una de las comunidades colindantes con el Parque Nacional Villarrica. 
no mapuche consideran beneficiosa la construcción e indican que atraerá más turistas y que si cruza por el parque los visitantes de Pucón pueden venir a este lado (Coñaripe) más fácilmente. A pesar de las contradicciones en la posición de estas personas hay una coincidencia y es que no quieren intervención del parque, con el corte de araucarias ${ }^{7}$, dado el valor sagrado que tiene para la cultura mapuche, valor que los colonos saben reconocer.

Con excepción de algunos proyectos de turismo comunitario y economía solidaria8 que se implementan en la zona, los enfoques dominantes siguen invisibilizando el significado del territorio para estas comunidades indígenas, marginando nuevamente sus necesidades culturales, sociales y económicas. La racionalidad sobre la que se sustenta el desarrollo como crecimiento económico no es compatible con la racionalidad de resguardo territorial propia de los pueblos originarios, a quienes se les continúa despojando sus territorios. Es así, como el extractivismo simbólico-paisajístico toma forma y socava las bases materiales sobre la que se sustenta la existencia del pueblo mapuche.

Hasta aquí puede afirmarse, que las corrientes ecologistas señaladas, confluyen en territorios ancestrales como nuevas formas de colonización, tanto de los pueblos originarios como de la naturaleza, primero, como ya se dijo, separando ser humanonaturaleza mediante el desplazamiento de los pueblos a zonas de amortiguación y; segundo, mediante la modernización de los territorios ancestrales para convertirlos en "mercancía verde".

${ }^{7}$ Las araucarias son coníferas de donde se recolecta el piñón, fruto relevante en la culinaria mapuche. Es un árbol sagrado para esta cultura.

${ }^{8}$ El enfoque de estos proyectos de turismo comunitario, facilita la definición de acciones desde las mismas comunidades respetando su cosmovisión, además generando condiciones para un desarrollo endógeno. Algunos de los proyectos con esta orientación son "Programa de Transferencia Tecnológica (PDT), Turismo de Intereses Especiales (TIE) y desarrollo con base local en Destino Sietelagos", ver página oficial http://trawun.cl/ ; y el "Nodo de Turismo Comunitario", ver página oficial http://melikofelen.cl/ 
Revista de Ciencias Sociales y Humanidades

\section{c. El ecosocialismo ¿una alternativa de reapropiación territorial?}

El ecosocialismo es una "corriente de pensamiento y de acción ecológica que hace propios los conocimientos fundamentales del marxismo" (Lowy, 2011). Este abordaje teórico se ocupa del análisis de conflictos ambientales surgidos por las dinámicas expansionistas y conservacionistas. A diferencia de otras corrientes como la ecología profunda y la ecología integral, el ecosocialismo es antropocéntrico, se mueve en el ámbito del materialismo histórico, sin embargo con una posición revisionista. Según Lowy:

[...] el ecosocialismo está fundado en una apuesta, que ya era la de Marx: la predominancia, en una sociedad sin clases, del "ser" por sobre el "tener", es decir, la realización personal de las actividades culturales, políticas, lúdicas, eróticas, deportivas, artísticas, políticas (sic), antes que la acumulación de bienes y de productos (2011, pp. 35-36).

Frente a las corrientes señaladas en los apartados anteriores, que establecen una racionalidad occidental donde se fracciona y monetariza la naturaleza, racionalidad fundada sobre el despojo de territorios ancestrales de las comunidades mapuche, el planteamiento de Lowy en relación con el "ser" sobre el "tener", brinda luces sobre la importancia de recuperar prácticas de intercambio que se resisten a morir en algunas comunidades indígenas. En las comunidades localizadas al sur del Parque Nacional Villarrica, subsisten prácticas como las "mingas", que además de generar trabajo colectivo, promueve valores de cooperación y transforma el valor de cambio del trabajo hacia el valor de uso, constituyéndose un espacio para el ocio, el conocimiento, el afecto, etc. Precisamente en la comunidad Carlos Antimilla se construyó con la colaboración de personas de la localidad y externas un "fogón del conocimiento" mediante la práctica de "la minga". El objetivo de este fogón fue posibilitar mediante transmisión oral el intercambio de conocimientos ancestrales (B. H, comunicación personal, 23 de mayo de 2015). Tanto en el proceso de construcción del fogón como en su objetivo, no media el valor de cambio, sino por 
el contrario, la organización está buscando recuperar prácticas que desmercantilicen las relaciones en la comunidad.

Pero también, al interior del territorio constituido como Parque, la práctica ya mencionada de las veraneadas favorecía en el pasado el valor de uso, dado que ni la buena nutrición de los animales, ni la fertilización de las tierras dependían del consumo de bienes disponibles en el mercado, sino que formaban parte de los bienes que la naturaleza tenía disponibles; también se generaban sinergias con otras necesidades humanas como lo es el conocimiento, el ocio y la identidad.

En este sentido, el desplazamiento de las comunidades mapuche ha implicado el despojo no solo material sino también cultural y económico, ya que se pasa de modos de satisfacer las necesidades básicas fuera del mercado a la generación de prácticas cotidianas que se actualizan con la moneda. De ahí la importancia del reconocimiento que hace el ecosocialismo al valor de uso y no al valor de cambio.

Ahora bien, surge el cuestionamiento sobre la sustentabilidad de estas prácticas ya que se han ejercido en espacios hoy destinados a la conservación. Siendo que por la cosmovisión de las comunidades mapuche, se está buscando cambiar de prácticas de conservación hacia un resguardo de ese territorio, haciendo "uso del espacio, pero de forma ancestral, sustentable, un uso espiritual, y los espacios que ahí existen, se mantengan, no sean alterados" (Chocorí, B, comunicación personal, 23 de abril de 2015). Chocorí señala además que ahora "las tierras están sobreexplotadas, erosionadas y las cuencas de agua están sin árboles, entonces hay que practicar las cosas antiguas, que hoy tienen más vigencia que nunca". Precisamente esta sobreexplotación tiene su explicación en las dinámicas del mercado y los hábitos de consumo de las sociedades biocéntricas, que están presionando los campos a fin de sostener una economía en constante crecimiento, sobre la base de los bienes de la naturaleza.

En este sentido, el ecosocialismo coincide con la ecología integral al plantear la crítica al sistema capitalista mundial. Pero, desconfiados de las posibilidades 
Revista de Ciencias Sociales y Humanidades

tecnológicas para detener el deterioro ambiental, los ecosocialistas consideran que no es posible un "capitalismo verde" ya que la expansión de este sistema lleva inherente la idea de crecimiento ilimitado, sin considerar las capacidades de la biósfera. Proponen, según Riechman, una economía homeostática tendiente "a buscar lo suficiente en vez de perseguir siempre más" (Riechman, 2013: p. 4). Se identifica en esta propuesta la posibilidad de sobrevivir de las comunidades tradicionales, en sus dimensiones materiales y simbólicas, constituyéndose en herramienta de lucha para alcanzar la justicia distributiva como valor de la dignidad humana (Riechman, 2001: pp. 226-227), entendida como

[...] acceso a los bienes y derechos mínimos necesarios para una existencia decente. La satisfacción de necesidades básicas como el alimento, el agua limpia, la salud y el alojamiento - y tal satisfacción, en el caso de las poblaciones más pobres del planeta que viven dentro de economías de subsistencia, tiene mucho que ver con el acceso directo a bienes ambientales- ... (Riechman, 2001, p. 226).

La propuesta de una economía homeostática hace posible la construcción de una sociedad más justa social y ambientalmente, anticapitalista que respete la diversidad cultural y posibilite la existencia del bien común y los bienes comunes, tal como lo propone (Riechman, 2013).

Al poner en perspectiva esta propuesta teórica frente a las presiones que enfrentan las comunidades mapuche estudiadas, es patente la influencia de los movimientos sociales indígenas que vienen posicionándose en las últimas tres décadas con demandas por la reapropiación de sus territorios, su autodeterminación y el respeto a sus modos de vida, en este sentido se tornan ambientalistas, pues, trascienden las demandas tradicionales para defender su cultura, sus modos de vida que se encuentran vinculados con la naturaleza (Leff, 1995). 
Precisamente, la búsqueda de reapropiación de los territorios tiene su referente en la idea de que sin territorio no se puede hacer ejercicio de la cosmovisión mapuche, porque cada uno de los elementos presentes tiene un significado dentro de su forma de ver el mundo (Chocorí, B., comunicación personal, 23 de abril de 2015). De ahí, que la re-apropiación del Parque Nacional Villarrica se constituya un tema de justicia ambiental, en tanto la naturaleza es fundamental para la sobrevivencia cultural de las comunidades tradicionales.

De esta forma el mismo Leff rescata las posibilidades de construcción de estilos alternativos de desarrollo, mediante la reapropiación de la naturaleza que plantea un principio de equidad en la diversidad. La lucha de comunidades rurales, campesinas, tradicionales implica

[...] la autodeterminación de las necesidades, la autogestión del potencial ecológico de cada región en estilos alternativos de desarrollo y la autonomía cultural de cada comunidad. Estos procesos definen las condiciones de producción y las formas de vida de diversos grupos de la población en relación con el manejo sustentable de su ambiente (Leff, 2004, p. 410).

Orientados por contar con espacios alternativos de existencia de su cultura, las comunidades mapuche aledañas al Parque Nacional Villarrica vienen construyendo proyectos de recuperación territorial. Ésta pasa por diferentes ámbitos, desde la definición de acuerdos con la administración del parque para hacer uso de su biodiversidad a partir de las prácticas tradicionales, hasta la recuperación de la toponimia. En el primer caso, en el año 2013, se diseñó en conjunto con los administradores del parque un "decálogo del piñonero" que pretendía posibilitar el ingreso de personas mapuche a recolectar el piñón utilizando prácticas sustentables; así establecen cuotas de extracción, reglas de ingreso al lugar, y formas de compensación mediante la siembra de una parte de lo recolectado (A. N. comunicación personal, 19 de mayo de 2015). Aunque este documento no se vio implementado por cambios en la división administrativa de las regiones, la 
Revista de Ciencias Sociales y Humanidades

experiencia comunitaria desarrollada en la construcción del decálogo, deja patente lo significativo de la práctica y la necesidad del territorio para recrearla.

Asimismo, con la pretensión de recuperar simbólicamente el territorio, a través de la enunciación de cada lugar desde su idioma, el mapusungún, han diseñado un proyecto de construcción de rótulos con los nombres originarios de cada lugar, que sustituyan los actuales tanto en la ciudad como en el Parque Nacional Villarrica, de tal forma que el idioma sea un vehículo para recuperar su "identidad" y se facilite la transmisión de saberes (B. H., comunicación personal, 23 de mayo de 2015). Es decir, recobrar la toponimia es percibida como una forma de reapropiarse del territorio, una reivindicación política desde el saber mapuche, donde cambiar un nombre como Coñaripe, así llamado uno de los poblados actualmente, y devolverle su nombre originario Conarrupu -que significa senda del noble, un noble que es un líder espiritual dominante de diferentes elementos- tiene una importancia trascendental tanto política como cultural.

\section{Conclusiones}

De la exploración realizada hasta aquí sobre las principales corrientes ecologistas y su relación con el conflicto socio-ambiental de las comunidades mapuche que habitan en las zonas de amortiguación del Parque Nacional Villarrica y el acceso a esta unidad de conservación, se desprende que el ecosocialismo aporta algunos referente para su lectura. Contrariamente, la ecología profunda, podría decirse, está en el origen del problema dado su interés eco-céntrico que urgió de la separación del ser humano y la naturaleza para poder preservarla, despojando así de los derechos ancestrales sobre los territorios de comunidades mapuche.

Por su parte, la ecología integral si bien hace una crítica al sistema capitalista, en el caso del Parque Nacional Villarrica, se propicia la modernización de los espacios para que a través del aumento de la visitación se financie la conservación y se propicie el desarrollo de las áreas de amortiguamiento, pero no plantea 
transformaciones profundas en los modos de relacionarse con la naturaleza, que permitan el uso ancestral del territorio.

El ecosocialismo sin embargo, en su crítica al capitalismo propone soluciones que buscan mejorar la calidad de vida de las personas y en específico de comunidades indígenas inmersas en conflictos socio-ambientales generados por la disputa de territorios, colocando la discusión en las transformaciones sobre el valor de uso y el valor de cambio, además se propone construir una economía homeostática, que posibilite su existencia. Una existencia donde prevalezca el valor de uso valoraciones sobre las araucarias como árbol sagrado y su fruto el piñón, las mingas como modo de vida que cambia formas de producción y es un espacio de socialización, las veraneadas y la recolección del piñón- esto es posible solo mediante la reapropiación territorial que permita la autodeterminación, la autogestión, la autodependencia resguardando la naturaleza.

\section{Bibliografía}

Aylwin, J. (7.12.2008). ¿De quién es el Parque Nacional Villarrica? www.lanacion.cl. Obtenido ww.lanacion.cl/prontus_noticias_v2/site/artic/20081206/pags/2008120621370 5.html

Corporación Nacional Forestal. (2006). Plan Nacional de Manejo Parque Nacional Villarrica. Obtenido de http://www.conaf.cl/wpcontent/files_mf/1382466395PNVillarica.pdf

Del Viso, N. (2011). Mecanismos comunitarios de resolución de conflictos socioecológicos. Una mirada sobre los aspectos culturales. En S. Álvarez (Ed.), Convivir para perdurar. Conflictos ecosociales y sabidurías ecológicas. España: Icaria Editorial.

Gobierno de Chile. (2013). Parque Nacional Villarrica. Plan Maestro 2013. Desarrollo Sustentable en Áreas Silvestres Protegidas. Chile. 
Revista de Ciencias Sociales y Humanidades

Gudynas, E. (2003). Ecología, economía y ética del desarrollo. Uruguay: CLAES.

Guerrero. (2010). Corazonar, una antropología comprometida con la vida. Miradas otras desde Abya-Yala para la Descolonización del Poder, del Saber y del Ser. Quito, Ecuador: Universidad Politécnica Salesiana.

Leff, E. (1995). ¿De quién es la naturaleza? Sobre la reapropiación social de los recursos naturales. Gaceta Ecológica (37), 28-35.

Leff, E. (2004). Racionalidad ambiental. La reapropiación social de la naturaleza. Buenos Aires, Argentina: Siblo XXI.

Leff, E. (2014). La apuesta por la vida. Imaginación sociológica e imaginarios sociales en los territorios ambientales del sur. México: Siglo XXI Editores.

Lowy, M. (2011). Ecosocialismo. La alternativa radical a la catástrofe ecológica capitalista. Argentina: El colectivo.

Martinez-Alier, J. (2014). El ecologismo de los pobres. Conflictos ambientales y lenguajes de valoración. Chile: Quimantú.

Naess, A. (1973). Los movimientos de la ecología superficial y la ecología profunda: un resumen. Ética ambiental para la conservación biocultural, 1-5.

Riechman, J. (2001). Un mundo vulnerable. Ensayos sobre ecología, ética y tecnociencia. España: Catarata.

Riechman, J. (2013). Para una caracterización del ecosocialismo en diez rasgos. Crisis civilizatoria y ecosocialismo por Michael Löwy. Madrid, España: FUHEM.

Ulloa, A. (2013). Controlando la naturaleza: ambientalismo transnacional y negociaciones locales en torno al cambio climático en territorios indígenas en Colombia. Revista Iberoamericana (XIII), 117-133. 